\title{
De Berlin à Orsay via Mexico
}

Dans ce texte, qui reprend une intervention orale effectuée au Théâtre de la Reine Blanche le 8 juin 2016, le physicien nucléaire Pierre Radvanyi nous raconte son « Odyssée ", qui l'a conduit d'Allemagne en France puis au Mexique lors des événements dramatiques de l'accession au pouvoir du régime nazi puis de la Seconde Guerre mondiale, et enfin, après la guerre, au laboratoire de Frédéric Joliot-Curie, à Paris puis à Orsay. Il a relaté ses souvenirs de cette période dans Au-delà du fleuve, avec Anna Seghers (Le Temps des Cerises, 2014).

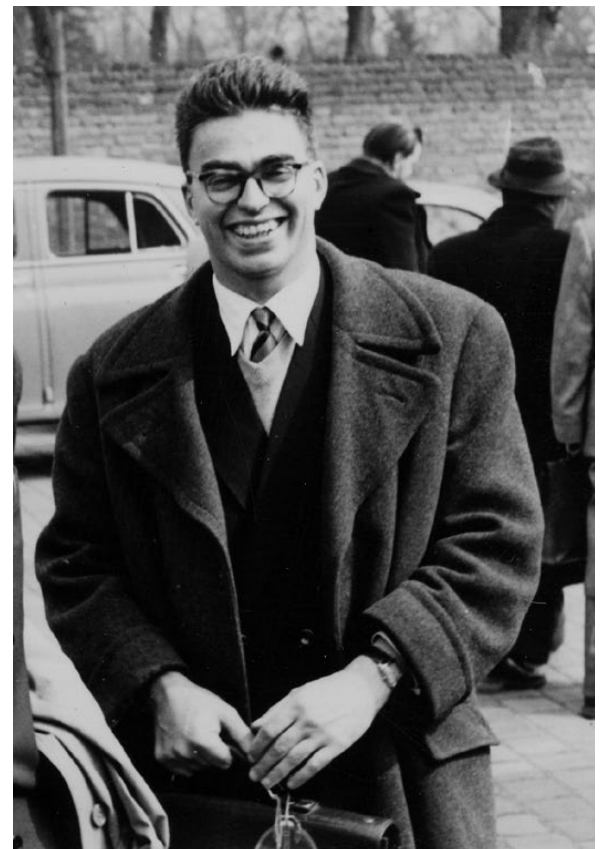

Pierre Radvanyi en 1956.

\section{"[Marietta Blau] me dit alors :}

"C'est un effet de la radioactivité ; comme chez les êtres vivants, il $y$ a des éléments chimiques qui naissent et meurent." Cette phrase m'impressionna durablement."
Je suis né à Berlin-Charlottenburg le 29 avril 1926. Ma mère, née à Mayence, avait commencé à écrire des nouvelles ; mon père, né à Budapest, était sociologue et économiste, il aimait enseigner. Mes parents avaient fait connaissance lors de leurs études à l'Université de Heidelberg. En 1928 parut le premier roman de ma mère - sous le pseudonyme d'Anna Seghers - qui lui valut le prix Kleist. Elle devint un écrivain engagé prenant position contre les nazis.

En 1932, à Berlin-Zehlendorf, j'apprends à lire et à écrire (en allemand, bien sûr), mais en décembre je suis victime d'une forte scarlatine. Le médecin recommande un séjour dans un home de convalescence en Forêt noire. Ma mère m'accompagne. En fin d'après-midi, le 27 février 1933, nous sommes sur le bord d'une patinoire lorsque les haut-parleurs donnent soudain une information : le Reichstag brûle ! Ma mère comprit tout de suite la signification de l'événement et rentra aussitôt à Berlin par le train de nuit. Peu après son arrivée dans notre appartement, on frappa à la porte : c'était la police venue l'arrêter pour ses prises de position antinazies ; la mère d'un voisin, membre des SA (organisation paramilitaire du parti national-socialiste), l'avait dénoncée. Elle est emmenée pour interrogatoire. Mais la police suivait encore les lois d'avant Hitler : constatant que ma mère était mariée à un Hongrois, elle l'autorisa à rentrer chez elle, tout en demeurant "à sa disposition ". Ma mère s'enfuit par la porte de derrière de notre appartement ; elle retrouva mon père et gagna Zürich. En avril ils émigrèrent à Paris.

Ma grand-mère de Mayence vint me chercher dans la Forêt noire début juin, pour m'emmener, avec ma sœur, en train, à Strasbourg, où ma mère nous attendait sur le quai. Ce fut la dernière fois que je vis ma grand-mère : elle fut déportée en mars 1942.

En France nous nous installâmes à Meudon ; c'est là que j'appris à parler français. Ma mère écrivait beaucoup, notamment son roman Das siebte Kreuz (La septième croix) en 1938/39. Avec ma sœur nous allâmes à "l'École nouvelle " (qui utilisait la méthode Freinet) ; j’y fis la connaissance, à onze ans, de Michel Langevin et de Christophe Tzara.

La guerre arriva en septembre 1939. Mon père fut interné comme Hongrois en mai 1940 au camp du Vernet d'Ariège. En juin 1940 ma mère décida de fuir avec ma sœur et moi pour tenter de "franchir la Loire ". Nous nous retrouvâmes sur les routes de l'exode avec des dizaines de milliers de réfugiés. Épuisés, sans nourriture, après une nuit de marche pour échapper à l'encerclement, après avoir dépassé Pithiviers, nous nous jetâmes sur des matelas dans une maison abandonnée par les paysans. Là, la Wehrmacht nous rattrapa et nous dépassa. Ce fut ensuite le retour forcé fin juin dans Paris occupé et, en septembre, la traversée clandestine de la ligne de démarcation. Trois mois à Pamiers dans l'Ariège, près du camp du Vernet, puis trois mois à Marseille. Chaque fois, ma mère m'envoya au lycée pour que je ne perde rien du programme !

Enfin, grâce aux visas mexicains délivrés par le consul général du Mexique, Gilberto Bosques, et à l'aide financière de la Ligue des écrivains américains, nous partîmes, le 24 mars 1941, à fond de cale, avec 250 autres réfugiés, sur un cargo, le Capitaine Paul Lemerle, pour la Martinique. À l'arrivée dans la baie de Fort-de-France, nous sommes internés plusieurs semaines à $\mathrm{La}$ Pointe du Bout. Finalement, au bout de plus de trois mois, après diverses tribulations, 

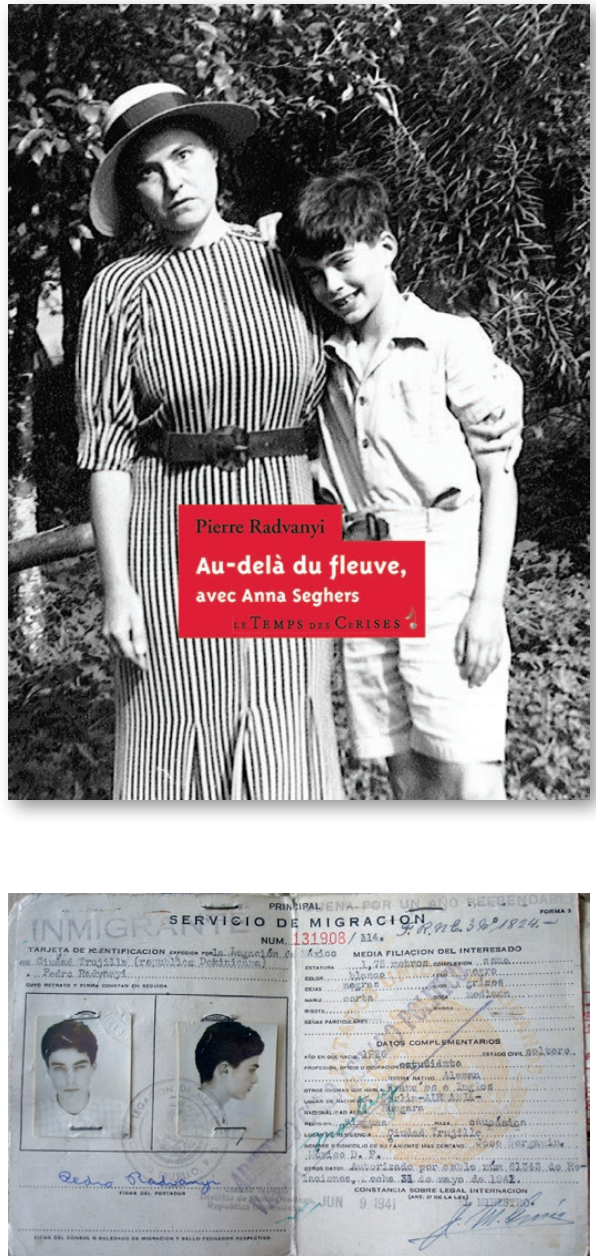

Carte d'immigration au Mexique (avec le tampon " admis comme réfugié politique »). dont un séjour à Ellis Island, nous arrivâmes, admis comme réfugiés politiques, à Vera Cruz au Mexique. Le roman de ma mère La septième croix, publié d'abord à Boston en traduction anglaise, connut immédiatement aux États-Unis un remarquable succès ; un film (avec Spencer Tracy dans le rôle principal) en fut tiré.

Je soutins mon baccalauréat français au lycée français de Mexico. J'avais choisi la section "math-élem ", mais le proviseur, ne disposant pas des professeurs nécessaires, m'avait engagé à trouver les enseignants correspondants à l'extérieur du lycée. J'eus ainsi comme enseignante pour la physique et la chimie Marietta Blau, une ancienne de l'Institut du Radium de Vienne, exilée au Mexique. Lors d'une de ses premières leçons, elle me montra sur son bureau une chambre d'ionisation et un morceau de minerai d'uranium ; je pus ainsi constater avec étonnement le déplacement des feuilles d'or ; elle me dit alors : "C'est un effet de la radioactivité ; comme chez les êtres vivants, il y a des éléments chimiques qui naissent et qui meurent. » Cette phrase m'impressionna durablement. Le président du jury du baccalauréat, l'ethnologue Paul Rivet, nommé par le général de Gaulle attaché culturel à Mexico, obtint pour moi une bourse pour faire, dès la fin de la guerre, mes études à Paris.
Lorsque j'acceptai cette proposition, ma mère me dit : "Pierre, je comprends parfaitement ton désir de retourner en France dès que possible et je t'approuve. Mais tu dois comprendre également que, pour ma part, c'est en Allemagne que je veux retourner, car un écrivain ne peut pas vivre et travailler longtemps en dehors du pays où l'on parle sa langue natale. » Muni des visas nécessaires, je quittai ma famille vers la fin septembre de 1945 et pris le train de Mexico à New York par St Louis. Un "Liberty ship" me mena au Havre, à moitié détruit, où j'arrivai le 16 octobre, dans un port partiellement reconstruit par l'U.S. Navy.

Le lendemain j'arrivai dans la matinée à la gare de Paris-Saint-Lazare. Je fis les démarches nécessaires pour ma bourse, mais il n'y avait personne pour me conseiller pour des études scientifiques. Je m'inscrivis donc aussitôt à la Faculté des Sciences. Intéressé par le phénomène de radioactivité, j'allais suivre par la suite le cours d'Irène Joliot-Curie à l'Institut du Radium. C'est ainsi que j'ai rejoint plus tard (en 1948, comme stagiaire de recherche CNRS) le laboratoire de chimie nucléaire de Frédéric Joliot-Curie au Collège de France.

Et c'est en 1957, trois ans après ma thèse, que je suis venu travailler dans le nouveau laboratoire construit sur le campus d'Orsay par Frédéric et Irène Joliot-Curie.

Pierre Radvanyi

\section{La carrière scientifique de Pierre Radvanyi}

Pierre Radvanyi, physicien nucléaire et historien des sciences, est directeur de recherche honoraire au CNRS.

En 1948, il entre au laboratoire de chimie nucléaire de Frédéric Joliot-Curie au Collège de France et prépare sa thèse de doctorat sous sa direction. Il est recruté comme chercheur par le CNRS et y fera toute sa carrière, de stagiaire de recherche à directeur de recherche émérite. Après la soutenance de sa thèse en 1954, il vient travailler en 1957 dans le nouveau laboratoire construit à Orsay par Irène et Frédéric Joliot-Curie. Ses domaines de recherche sont successivement les phénomènes de radioactivité, les réactions nucléaires, la production de particules élémentaires dans les noyaux. Il est auteur ou coauteur de nombreuses publications scientifiques, ainsi que d'articles et de livres de vulgarisation et d'histoire des sciences.

II est l'un des fondateurs du laboratoire national Saturne (IN2P3/ CNRS - CEA) à Saclay. Il organise plusieurs colloques et congrès de physique français et internationaux.

Pierre Radvanyi est docteur honoris causa de l'Université d'Uppsala (1995). Il est secrétaire général de l'association Curie et Joliot-Curie et poursuit actuellement son activité en histoire des sciences à l'Institut de physique nucléaire de l'Université Paris-Sud à Orsay.
-1978-1985 : Directeur-adjoint du Laboratoire National Saturne

• 1975-1980 : Secrétaire général de la Société Française de Physique

-1970-1997 : Rédacteur en chef du Bulletin de la SFP

• 1976-1981 : Membre du Comité exécutif de la Société européenne de Physique

-1985-1994 : Chargé de mission à la direction des relations internationales du CNRS

Livres de Pierre Radvanyi

- La radioactivité artificielle et son histoire

(avec M. Bordry), Éditions du Seuil (1984)

- Histoires d'atomes (avec M. Bordry), Éditions Belin (1988)

- Euvre et engagement de Frédéric Joliot-Curie

(éditeur, avec M. Bordry), EDP Sciences (2001)

- Les rayonnements nucléaires, collection Que sais-je?,

Presses Universitaires de France (1995)

- Les Curie, pionniers de l'atome, Belin (2005)

- Histoire de l'atome, de l'intuition à la réalité, Belin (2007) 